\title{
Development of a CCD-Based Image Acquiring System for Neutrons at the Argonauta Reactor
}

\author{
Marcelo J. Gonçalves, Ricardo Tadeu Lopes, \\ Universidade Federal do Rio de Janeiro - COPPE - Bloco 6, 21945-970, Rio de Janeiro, RJ, Brazil \\ Maria Ines Silvani, Gevaldo L. de Almeida, and Rosanne C. A. A. Furieri \\ Instituto de Engenharia Nuclear - CNEN - C.P. 68550, \\ Ilha do Fundão, 21945-970, Rio de Janeiro, RJ, Brazil
}

Received on 6 July, 2005

\begin{abstract}
A thermal neutron image acquiring system is being developed at the Instituto de Engenharia Nuclear-IEN $(C N E N)$. This system would be capable to perform non-destructive assays after $2 \mathrm{D}$ images acquired on a real or quasi real time basis as well as $3 \mathrm{D}$ tomographic images reconstructed from the two-dimensional ones. The source-detector set is constituted by the Argonauta reactor - furnishing a thermal neutron flux of $4.46 \times 10^{5} n$. $\mathrm{cm}^{-2} \cdot \mathrm{s}^{-1}$ - and by a converter-scintillating screen for this kind of radiation. After a $90^{\circ}$ deviation from its original path through a dark chamber provided with a properly positioned mirror, the light emitted by this screen is captured by a CCD-based video-camera coupled to a microcomputer which processes the data and supplies the final digital images. As a previous step aiming at the gathering of parameters and features to characterize the system, a conventional photo camera has been used instead of the video-camera being yet purchased. This work presents the qualitative and quantitative results obtained by using this preliminary system, such as $2 \mathrm{D}$ images of several objects and the Modulation Transfer Function-MTF respectively. Besides that, a comparison is made between the results achieved by using this system and those arising from a traditional neutrongraphy employing radiographic films.
\end{abstract}

\section{INTRODUCTION}

The different attenuation coefficients of a material for thermal neutrons and X-rays make these radiations complementary tools for non-destructive assays. Indeed, samples containing different material could be utterly transparent or opaque to one kind of radiation but would reveal its inner features to the other kind. Such features motivated the development of 2D tomographic systems incorporating conventional as well as position sensitive detectors and the Argonauta reactor as source of thermal neutrons [1,2]. Although conventional neutrongraphy using radiographic films exhibit better resolution, they only cast a kind of shadow of the attenuating materials inside the sample, but not a slice of it as a tomography does. However, a price in terms of acquisition time has to be paid for such a refined information: while a neutrongraphy carried out under the available thermal neutron flux of $10^{5} \mathrm{n} \cdot \mathrm{cm}^{-2} \cdot \mathrm{s}^{-1}$ requires about $40 \mathrm{~min}$, the tomography demands 2 to 6 hours depending on the type of the system employed. The aim of the present work is the development of a system capable to acquire neutrongraphic images of an object on a real time basis and the processing through a proper unfolding software of the images taken at different angles to reconstruct a $3 \mathrm{D}$ attenuation map the object being inspected. This system uses a $2 \mathrm{D}$ detector constituted by a screen containing a scintillating material capable to transform the energy of the incident radiation into visible light, and a proper camera to catch that light. Employing a video-camera it would be possible to acquire a sequential set of frames within a short time making thus feasible to assay dynamic structures. Since the neutrons themselves due to their lack of electric charge cannot undertake any ionization, the scintillating screen should contain a material capable to translate their presence into an ionization event, through a proper nuclear reaction. Materials containing isotopes such as ${ }^{6} \mathrm{Li},{ }^{10} \mathrm{~B},{ }^{155} \mathrm{Gd},{ }^{157} \mathrm{Gd}$ are widely employed for this purpose. Some basic concepts, as well as the methodology and results achieved in the first step of this work aiming at the development of a $5^{\text {th }}$ generation tomographic system is presented as follows. For the sake of characterization of the system, parameters and images of some objects have been obtained by using a conventional $50 \mathrm{~mm} / \mathrm{f} 1.8,35 \mathrm{~mm}$ photo-camera instead of the ultimate video-camera being yet purchased. The $15 \times 10 \mathrm{~cm}$ scintillating screen is constituted by silver-activated $\mathrm{Li}^{6} \mathrm{~F}(\mathrm{ZnS})$, being commercially available as NE426.

\section{SCINTILLATION MECHANISM}

The ${ }^{6} \mathrm{Li}$ in the scintillating screen is the responsible for the neutron-to-charged particle conversion through the reaction ${ }^{6} \mathrm{Li}+\mathrm{n}={ }^{3} \mathrm{H}+\alpha+4,78 \mathrm{MeV}$. The changing electrical field created by the moving charged reaction products displaces electrons in the crystal lattice from their valence band to the conduction band. When they return to their former level, the energy absorbed in that jump is given back as electromagnetic radiation. Since the energy gap of this forbidden zone between those bands lies outside the visible range, it is necessary to apply a trick to overcome this constraint. This is done by adding an activator to create intermediate energy levels within the otherwise forbidden zone, allowing the electrons in the conduction band to loose their energy in a partial fashion along their journey towards the valence band by jumping between those levels. These small jumps yield radiation 
within the visible range [3], as outlined in the Fig.1.



FIG. 1: The energy loss of the electron in a stepwise fashion rather than a single jump, allows the emission of radiation within the visible range.

\section{MODULATION TRANSFER FUNCTION}

The Modulation Transfer Function-MTF is a tool used to quantify the quality of the image furnished by an image acquiring system. This function shows how the ability of the system to distinguish single features is impaired by the gap between them. This is done by plotting the modulation, defined as the ratio of amplitude-to-mean value, versus the spatial frequency. When this spatial frequency increases, the Line Spread Function-LSF representing the system resolution begin to overlap increasing the mean value and reducing the amplitude, as shown in Fig.2. As consequence, the modulation decreases as the frequency increases.

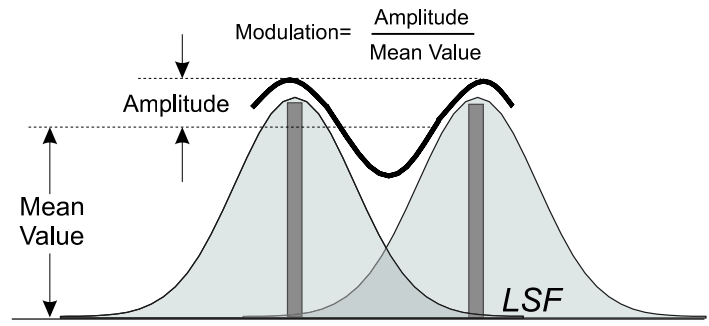

FIG. 2: The LSF representing the system resolution overlap at high frequencies diminishing thus the modulation.

The MTF can be obtained by the discrete Fourier Transform of the Line Spread Function-LSF [4] but it requires the knowledge of its profile what is not a readily achievable task. It can be also obtained by using a special collimator provided with constant aperture slits and increasing spatial frequency, but such collimators are very expensive or not available at all. In this work a simplified alternative of this approach has been employed by using several individual twin-slit collimators with different gaps to simulate a variable spatial frequency.

\section{EXPERIMENTAL AND RESULTS}

The NE 426 scintillating screen reacts to the incident neutrons by emission of visible light with a peak of intensity occurring at about $450 \mathrm{~nm}$, which corresponds to the bluishgreen light in the electromagnetic visible spectrum.

The image acquiring system as outlined in the Fig. 3 which is self-explainable, uses a dark chamber provided with a firstsurface mirror to deflect the light at $90^{\circ}$, precluding thus the direct incidence of radiation in the camera.

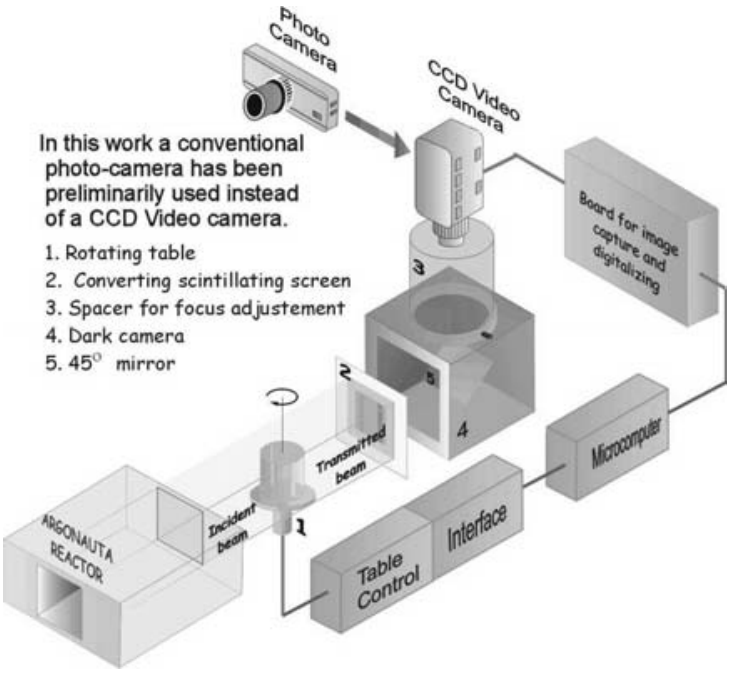

FIG. 3: Scheme of the image acquiring system. A conventional photo-camera has been preliminary used during the first phase of this work.

After the development of the film, the caught image is transformed through a proper digitalization into set of 3 matrices expressing the saturation for the basic colors, red, green and blue (RGB system). Since the main information concerning the beam attenuation is brought mainly by the blue matrix, the remaining ones can be ruled out without a substantial loss of quality in the final image.

The Modulation Transfer Function-MTF for the system has been measured aiming at the evaluation of the spatial resolution of the employed screen. The Fig.4a shows the experimentally obtained rough data used to construct the MTF shown in Fig.4b. Those data have been gathered by using three gadolinium masks, each of them provided with a pair of $1 \mathrm{~mm}$-width slits disposed at different gaps, defining thus different spatial frequencies. This collimator simulates approximately therefore the response of the system to two line sources, as an overlapping of two Line Spread Functions-LSF, which are specific for each system. The curves shown in Fig. 4a have been obtained by using the software MATLAB to digitalize the intensity of the blue tonality along a straight line perpendicular to the slits.

It is currently agreed that an image has an acceptable quality whenever the modulation surpasses a $10 \%$ cutoff value [5]. After the MTF for the system shown in Fig. 4b, that cutoff value corresponds to a spatial frequency of $1,67 \mathrm{~mm}^{-1}$, i.e. the system is capable to resolve features greater than $600 \mu \mathrm{m}$ 

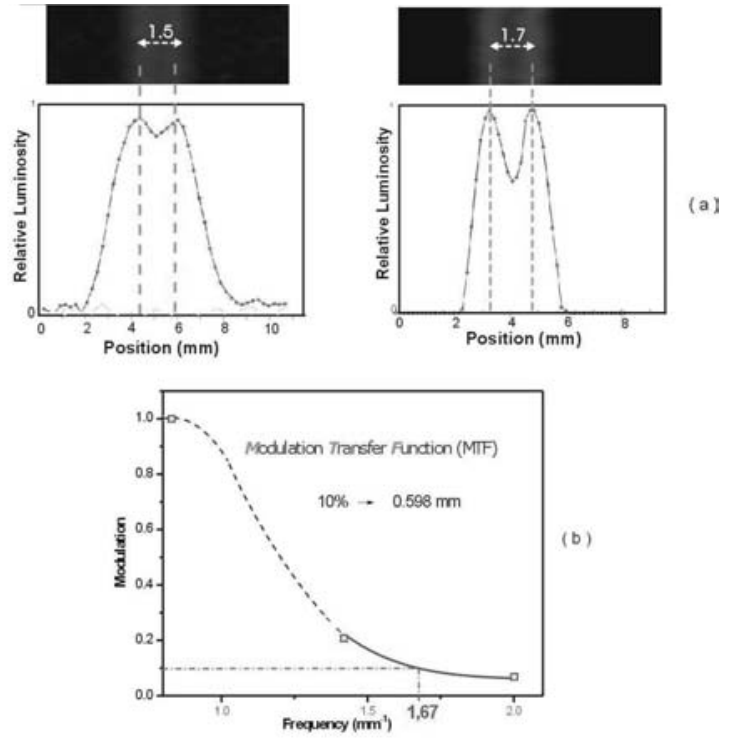

FIG. 4: Every point of the MTF was determined by the amplitudeto-mean value ratio from curves in (a).

approximately. These results have been compared with those ones obtained with the conventional neutrongraphy using radiographic films. Due to their intrinsic high resolution the related LSF for these films is very narrow and only begin to entangle at very high spatial frequencies which lies far beyond the range of the available collimators.

Some images of different objects as displayed by scintillating-converter screen and caught by a conventional photo-camera are shown in Fig.5-7.
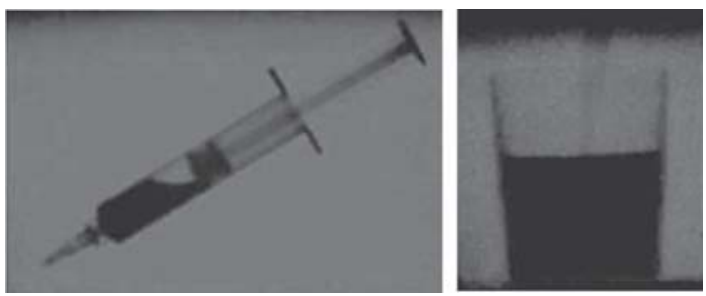

FIG. 5: Images after 30 seconds exposition time lapse. Water-filled plastic syringe containing an air bubble (left). Water-filled glass cup containing a stainless steel spoon (right).

The Fig.6 (top) shows the otherwise unknown inner structure of a automobile fuel injector gauge, revealing the presence of neutron-absorbing internal components as well external ones, such as rubber o-rings.

The Fig.6 (bottom) shows a ball-bearing where the voids between the balls formerly filled with grease appear as dark regions. The grease has later on been removed in order to take the conventional picture.

A neutrongraphic image of a cadmium foil provided with $0.5 \mathrm{~mm}$-diameter orifices placed at about $0.5 \mathrm{~mm}$ apart from each other is shown in Fig. 7. The system was unable to resolve the individual orifices which appear as somewhat con-
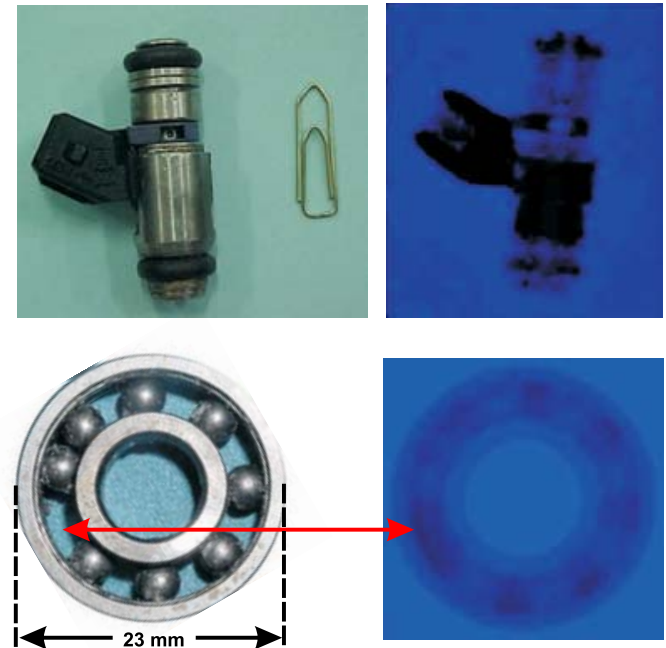

FIG. 6: (top) Automobile fuel injector. The rubber o-rings and plastic parts appear as dark areas. (bottom) Ball bearing. The grease between the balls (removed to take the conventional photo) appears as dark spots.

tinuous blurred stripe.
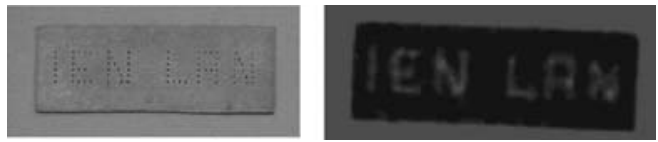

FIG. 7: Image of a cadmium foil provided with $0.5 \mathrm{~mm}$-diameter orifices.

Although the images acquired after the traditional neutron radiography using films exhibit a much better resolution, they demand a much high exposition time lapse. Moreover, the use of a video-camera would enable to acquire images of moving objects. Besides that, a set of digital images taken from an object under different angles, as provided by a video-camera, could be unfolded through a proper reconstructing algorithm to yield a 3D image. Such tasks are utterly impossible or very cumbersome to be accomplished for a system employing radiographic films. A brief qualitative comparison between the images furnished by the proposed system and the conventional neutrongraphy using radiographic films is shown in Fig.8 and 9.
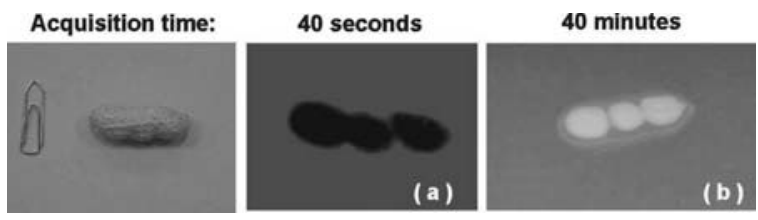

FIG. 8: (a) Neutrongraphy using a scintillating screen. (b) Conventional neutrongraphy.

The neutrongraphy of a plant "Scindapsus aureus"popularly known in Brazil as jiboia (Boa Constrictor) - obtained after the two above outlined approaches is presented in 
Fig. 9.
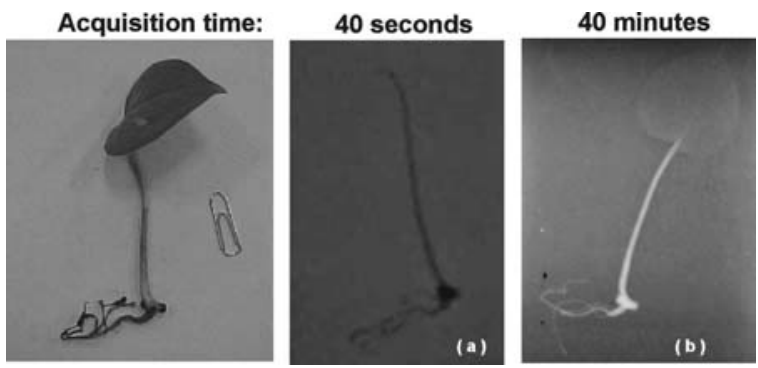

FIG. 9: (a) Neutrongraphy using a scintillating screen. (b) Conventional neutrongraphy.

\section{CONCLUSIONS}

The radiographic images acquired with thermal neutrons using a converter-scintillating screen as $2 \mathrm{D}$-detector demons- trated the feasibility to develop a 3D-tomographic system.

In spite of the low neutron flux available at the main channel of the Argonauta research reactor, the lack of an image intensifier, and a CCD-camera, this work has succeed in determining the Modulation Transfer Function - MTF for the system and to obtain fair quality images of different types of objects.

Both quantitative and qualitative results have been achieved by using a conventional photo-camera to catch the picture drawn on the converter-scintilllating screen. Further works will include the replace of that device by a CCD-camera, making thus possible a faster image acquisition, their storage in electronic form, and their processing by a proper image reconstructing software to obtain a final 3D image.

[1] M. I. Silvani, et al, Conversion of a X-ray position sensitive detector for use in a thermal neutron tomographic system, Nuclear Instruments and Methods in Physics Research B 213, 294 (2004).

[2] M. I. Silvani, et al, Evaluation of a Computer aided Neutron Tomografic System Incorporating a Gaseous Position Sensitive Detector, Nuclear Instruments and Methods in Physics Research A, 505, 568 (2004).

[3] F. G. Knoll, Radiation Detection and Measurement, ed. 2, USA, John Wiley and Sons(1989).

[4] ASTM E 1441-95 and 1570-95a, Non-Destructive Testing, Radiation Methods, Computed Tomograph. Guide for Imaging and Practice for Examination, ISO/TC 135/SC 5, N 118, USA (1996.)

[5] M. J. Gonçalves, Optimization of a Position Sensitive Detector for use in Tomographic Systems with Thermal Neutrons, M. Sc. Thesis, COPPE/UFRJ-Brazil 2003 (In Portuguese). 